\title{
El papel de las cofradías de pescadores de Galicia en la consecución de los Objetivos de Desarrollo Sostenible: protección de los recursos marinos y equidad
}

\section{Iria García Lorenzo}

RESUMEN: Las cofradías de pescadores son organizaciones regionales que agrupan a los pescadores de pequeña escala y mariscadores (en el caso de Galicia). Su papel dentro del sector pesquero les otorga competencias en la gestión y explotación de recursos marinos, actividades que realizan de modo colectivo. Esta posición permite a las cofradías influir en diferentes aspectos del desarrollo sostenible, principalmente en el nivel local.

Partiendo de casos de estudio anteriores, este trabajo busca avanzar en el papel que juegan las cofradías de pescadores de Galicia en la consecución de los Objetivos de Desarrollo Sostenible. En concreto, el análisis se centra en la protección de los recursos marinos, del objetivo de Vida Submarina, y en la consecución de la equidad, del objetivo de Reducción de las Desigualdades. Las funciones atribuidas dentro de la cogestión de los recursos marinos, su origen tradicional, su carácter autogestionario y el cumplimiento de los principios cooperativos son las principales características de las cofradías que pueden favorecer la implementación de estos Objetivos de Desarrollo Sostenible.

PALABRAS CLAVE: Cofradías de pescadores, Objetivos de Desarrollo Sostenible, gestión de recursos marinos, protección ambiental, carácter auto-gestionado, equidad.

CLAVES ECONLIT: D23, J54, L32, Q01, Q22.

Cómo citar este artículo/How to cite this article: GARCÍA, I. (2021): "El papel de las cofradías de pescadores de Galicia en la consecución de los Objetivos de Desarrollo Sostenible: protección de los recursos marinos y equidad", CIRIEC-España, Revista de Economía Pública, Social y Cooperativa, 102, 97-122. DOI: 10.7203/CIRIEC-E.102.18388.

Correspondencia: Iria García Lorenzo, Departamento de Economía Aplicada, ERENEA, ECOBAS, Universidad de Vigo, iriagarcia@uvigo.es. 


\section{Expanded abstract}

\section{The role of the Galician Cofradías in achieving the Sustainable Development Goals: protection of marine resources and equity}

\section{Contextualization and objectives}

In Galicia (northwest Spain), shellfish gatherers and small-scale fishers are organised in the socalled Cofradías, entities that arise from a long associative tradition (Taboada, 2004) and have a recognised position in the fishing sector. Given their historical adaptation and the existing regulatory framework, cofradías are entrusted with functions within a system of marine resources co-management (McGinnis and 0strom, 2014; Jentoft and Chuenpagdee, 2015), sharing responsibilities with the European, national and regional administrations (Law 3/2001; Regulation (EC) no 2371/2002; Decree 8/2014).

At the same time, cofradías of Galicia (and Spain) are recognised as Social Economy entities (Cervera, 2010; Law 5/2011; Law 6/2016). In Galicia there are 63 cofradías, which combine the tasks of the Public Administration with the development of productive and commercialisation activities (Decree 8/2014; Federación Galega de CP, 2018). These activities have a business nature and are autonomously managed by their members, who share and benefit from the use and exploitation of marine resources (Astorkiza et al, 2018; García-Lorenzo et al, 2019b). Thus, cofradías have a hybrid character between the public and the private (Williamson, 2007; Mc Ginnis and 0strom, 2014; Jentoft and Chuenpagdee, 2015) that conditions its organisational structure and its performance (García-Lorenzo et al, 2019a).

Their role within the fishing sector gives them competences in the management and exploitation of marine resources, activities that they carry out collectively as a Social Economy entities. This position allows cofradías to influence different aspects of sustainable development, mainly at the local level. In this way, the objective of this work is to study how the Galician cofradías contribute to the Sustainable Development Goals (SDG). Specifically, the study is focused on the protection of coastal marine and inland water resources, the equity in access to common goods and the income distribution, related to SDG 14 of Life Below Water and SDG 10 of Reduced Inequalities.

\section{Design and methodology}

The analysis is based on three previous studies about cofradías of Galicia. Two of them were done from the institutional perspective and focused on the adaptative processes in cofradías 
(García-Lorenzo et al, 2019a) and the analysis of governance interactions (García-Lorenzo and Varela-Lafuente, 2019). Another one was done from the perspective of the Social Economy and participatory firm approach and focused on members' participation in cofradías (García-Lorenzo et al, 2019b).

With these bases, the study aims to show a joint vision of the Galician cofradías as entities that simultaneously belong to two areas that, individually, are well positioned to contribute to sustainable development: small-scale fisheries (WorldFish et al, 2018; Pascual-Fernández et al, 2020; Said et al, 2020) and the Social Economy (Bastida et al, 2020; Castro-Nuñez et al, 2020; Chávez-Ávila and Gallego-Bono, 2020). The integrated analysis of this dual position seeks to deepen the capacity of Galician cofradías to contribute to the achievement of SDG 10 and SDG 14.

Consequently, the study is structured in four sections: 1) Introduction; 2) Presentation of the cofradías highlighting their position as actors within a fisheries co-management system and their configuration as Social Economy entities; 3) Analysis of cofradías' characteristics that contribute to the achievement of SDGs 10 and 14; 4) Presentation of the main conclusions of the study.

\section{Results, limitations and implications}

The analysis carried out allows to present the capacity of Galician cofradías to contribute to certain aspects of sustainable development. Thus, it is shown that the functions attributed within the co-management of marine resources, their traditional origin, their self-management character and compliance with cooperative principles are the main characteristics of cofradías that can favor the implementation of these Sustainable Development Goals.

Specifically, the results focus on the ability to contribute to SDG 14 of Life Below Water and some of its targets (14.2 - Sustainably manage and protect marine and coastal ecosystems; 14.4 - Effectively regulate harvesting and implementation of science-based management plans; 14.5 - Conserve at least 10 per cent of coastal and marine areas; $14 . b$ - Provide access for small-scale artisanal fishers to marine resources and markets; $14 . c$ - Enhance the conservation and sustainable use of oceans and their resources), and to SDG 10 of Reduced Inequalities and some of its targets (10.1- Achieve and sustain income growth of the bottom 40 per cent of the population at a rate higher than the national average; 10.2 - Empower and promote the social, economic and political inclusion of all; 10.3 - Ensure equal opportunity and reduce inequalities of outcome).

The study also presents certain synergies with other SDG, such as SDG 1 of No Poverty, SDG 2 of Zero Hunger, SDG 5 of Gender Equality, SDG 8 of Decent Work and Economic Growth or SDG 16 of Peace, Justice and Strong Institutions. However, the study does not carry out an exhaustive analysis of cofradías' capacity of influence all the SDGs.

This study helps to better understand how the actions of the Galician cofradías (and the definition of their regulatory framework) can generate synergies in multiple aspects of sustainable development, given their position in the small-scale fisheries sector and the social economy sector. 


\section{Conclusions and original contributions}

The originality of the study lies in interrelating the position and characteristics of the Galician cofradías with the Sustainable Development Goals. In this way, it is identified that Galician cofradías have an important capacity to influence in some aspects identified in the Agenda 2030, such as resources protection, access to natural and common resources and economic redistribution. This influence is possible mainly because of their situation as actors and users within the fishing and shellfish co-management system and the public-private nature of their organisational structure. This structure arises from the adaptation to the historical institutional conditions and the self-organised character, and has framed cofradías as Social Economy entities that comply with the cooperative principles.

Cofradías share the management of coastal and inland water resources with public administrations and other entities in the sector, jointly protecting them and contributing to the objective of Life Below Water. Specifically, they collaborate in the organisation of the sector and carry out control and surveillance tasks, as well as several sustainable management plans. To fulfill these tasks, the cofradías actively participate in decision-making processes of the fishing co-management system, although this participation is not always effective.

The link to the territory of these organisations and the collective way of exploitation also encourages the perception of the resource as a means of subsistence and provides resilience to the sector. Traditional production methods are maintained, making the activity less intensive and aggressive with the marine environment, and a closer and more real knowledge of the resource is achieved, obtaining useful information for fisheries management. Despite this, there are conservative and short-term attitudes among fishers, derived from the confluence of interests, which can go against sustainable development.

On the other hand, cofradías carry out their business activity in a local and rural environment, promoting the endogenous development of the region through the fixation of population and the generation of added value. This business activity is carried out collectively, democratically and complying with the principle of economic participation, which contributes to the achievement of the objective of Reduced Inequalities. At the same time, some characteristic of the cofradía as a Social Economy entity, contribute to the reduction of inequalities in the access to natural and common resources.

Keywords: Cofradías, Sustainable Development Goals, Marine resources management, environmental protection, self-management, equity. 


\section{Introducción'}

La Comunidad Autónoma de Galicia, en el noroeste de España, mantuvo históricamente una significativa dependencia de la pesca (Surís y Santiago, 2014). En la actualidad, el sector se encuentra dividido entre las actividades altamente industrializadas (pesca de altura y gran altura, granjas acuícolas) y la pesca a pequeña escala (pesca artesanal, costera y marisqueo). Estas pesquerías a pequeña escala son un sector significativo en el conjunto regional donde las embarcaciones y aparejos son propiedad de empresas familiares, dirigidas por una o pocas personas, que desarrollan procesos simples de explotación de los recursos y de bajo impacto ambiental (Comisión Europea, 2016). Su relevancia en la actual Agenda 2030 destaca en el Objetivo de Desarrollo Sostenible 14 de Vida Submarina, que busca la conservación y uso sostenible de los océanos, mares y recursos marinos, y menciona específicamente a la pesca artesanal entre sus metas (Resolución A/RES/70/1 de la Agenda 2030 para el Desarrollo Sostenible; WorldFish et al, 2018; Said y Chuenpagdee, 2019).

En Galicia, las mariscadoras, mariscadores y pescadores de pequeña escala se organizan en las llamadas Cofradías de Pescadores, entidades que provienen de una larga tradición asociativa (Taboada, 2004) y tienen una posición reconocida en el sector pesquero. Dada su adaptación histórica y el marco normativo existente, las cofradías tienen encomendadas funciones dentro de un sistema de cogestión de recursos marinos (McGinnis y 0strom, 2014; Jentoft y Chuenpagdee, 2015), compartiendo responsabilidades con los administraciones europeas, estatales y autonómicas (Ley 3/2001; Reglamento (CE) nº 2371/2002; Decreto 8/2014). Conseguir una explotación sostenible de la pesca a largo plazo y una protección ambiental del medio marino son algunas de estas responsabilidades.

Las cofradías de pescadores de Galicia y España son reconocidas como entidades de Economía Social (Cervera, 2010; Ley 5/2011; Ley 6/2016). En Galicia hay 63 cofradías, las cuales compaginan labores propias de la Administración Pública con el desarrollo de actividades productivas y de comercialización (Decreto 8/2014; Federación Galega de CP, 2018). Estas actividades tienen una naturaleza empresarial y son gestionadas de forma autónoma por sus miembros, quienes comparten y se benefician del uso y la explotación de los recursos marinos (Astorkiza et al, 2018; García-Lorenzo et al, 2019b). Así, las cofradías de pescadores tienen un carácter híbrido entre lo público y lo privado (Williamson, 2007; Mc Ginnis y 0strom, 2014; Jentoft y Chuenpagdee, 2015) que condiciona su estructura organizativa y su actuación (García-Lorenzo et al, 2019a).

En este contexto, el objetivo de este trabajo es estudiar cómo contribuyen las cofradías de pescadores de Galicia a los Objetivos de Desarrollo Sostenible (ODS). Para ello, en primer lugar, se presenta a las cofradías destacando su posición como actores dentro de un sistema de

1. Artículo aceptado como comunicación en XVIII Congreso Internacional de Investigadores en Economía Social y como documento de trabajo en el UNTFSSE: https://knowledgehub.unsse.org/knowledge-hub/el-papel-de-las-cofradias-de-pescadores-de-galicia-noroeste-de-espana-en-la-proteccion-de-los-recursos-marinos-y-consecucionde-la-equidad-ventajas-y-limitaciones/ 
co-gestión pesquera y su configuración como entidades de Economía Social. A continuación, se analizan las características propias de las cofradías que contribuyen y limitan la consecución de los ODS. Partiendo de casos de estudio anteriores, el análisis se centra en la protección y el acceso a recursos marinos, del objetivo de Vida Submarina, y en la consecución de la equidad en la distribución de ingresos, del objetivo de Reducción de las Desigualdades. El trabajo termina presentando las principales conclusiones derivadas.

\section{Las cofradías de pescadores de Galicia: entidades de Economía Social del sector pesquero}

Las cofradías de pescadores son las principales organizaciones representativas de los pescadores a pequeña escala y mariscadores de Galicia. Tienen un origen privado, como una agrupación espontánea y libre de armadores y marineros para la defensa de sus intereses comunes. La necesidad de una tener una protección mínima frente a los riesgos propios de la actividad pesquera, así como la incertidumbre en las capturas e ingresos, modelaron la organización de las personas relacionadas con esta actividad.

Esta tradición asociativa proviene de experiencias históricas de los siglos XI-XIII, siendo el gremio la primera forma organizativa conocida. Desde la abolición de los gremios en 1861, estas asociaciones han pasado por la forma de sociedad de socorro mutuo, pósito marítimo de pescadores y diferentes tipologías de cofradías. La intervención del Estado en su organización, característica de las cofradías actuales, fue un hecho posterior a su nacimiento (Taboada, 2004).

La evolución en su forma organizativa fue paralela a los avances pesqueros. El uso del arte de pesca de cerco favoreció la pesca auto-organizada y el trabajo en grupos formados por varias familias, donde se necesitaban hombres para la pesca y mujeres para la elaboración y el mantenimiento de las redes. La posterior aparición del arte de arrastre y los avances tecnológicos provocados tras la revolución industrial, como la máquina de vapor, transformaron el escenario. La explotación pesquera se convirtió en una actividad más intensiva en capital y agresiva con el medio marino y aparecieron dos sectores diferenciados: la pesca de bajura, artesanal o local y la pesca de altura. Mientras que la pesca artesanal tuvo menor nivel de desarrollo, manteniendo métodos tradicionales de producción y actuando en la zona costera, la pesca de altura recibió grandes inversiones y se convirtió en una actividad industrial (Labarta, 1979).

La explotación intensiva y extensiva del medio marino influyó en cómo la literatura económica trataba no solo a los recursos pesqueros, sino a todos los recursos naturales renovables. Así, tras la Segunda Guerra Mundial y la creciente preocupación por la sobreexplotación, los estudios de los recursos naturales renovables como objeto específico y conjunto de análisis, 
alcanzaron relieve. Desde entonces, en la pesca, estos estudios han girado principalmente en torno a dos grandes enfoques que buscan las mejores soluciones de gestión sostenible (Garza y Varela, 2005): el análisis bioeconómico (Gordon, 1954; Scott, 1955; Schaefer, 1957; Clark y Munro, 1975; Arnason, 1989, 1994) y la Nueva Economía Institucional (Schlager y 0strom, 1990, 1992; Mc Ginnis y Ostrom, 2014; Jentoft y Chuenpagdee, 2015; Linke y Bruckmeier, 2015).

Este desarrollo teórico se ha traducido en un marco internacional de gestión de los recursos marinos, aceptado por diferentes Estados y organismos internacionales y sustentado en la propiedad del medio natural y los derechos pesqueros (Stevenson, 1991). Desde la Convención de los Derechos del Mar de 1982, los Estados son soberanos y ostentan la propiedad de los recursos en sus Zonas Económicas Exclusivas. En el caso de España, la gestión de sus recursos marinos está determinada por otros dos factores: i) su pertenencia a la Unión Europea, que implica la asunción del marco comunitario; y ii) su organización territorial, que permite delegar algunas competencias de gestión en las Comunidades Autónomas, principalmente en aguas interiores (Reglamento ${ }^{\circ}$ 2371/2002; Ley 3/2001; Ley 11/2008; Ley 6/2009; Ley 33/2014; García-Lorenzo et al, 2019a).

En este contexto de diferentes sectores pesqueros y multitud de administraciones responsables de la gestión, la forma de asociación de los pescadores de pequeña escala se ha adaptado hasta conformarse las cofradías actuales. Las características básicas de las cofradías de España se establecen a nivel estatal y se definen, legalmente, como corporaciones de derecho público, sin ánimo de lucro, representativas de intereses económicos, que actúan como órganos de consulta y colaboración de las administraciones competentes en materia de pesca marítima y de ordenación del sector pesquero (Ley 3/2001). Esta definición como corporación de derecho público ha permitido a las Comunidades Autónomas asumir las competencias de su regulación a través de leyes de pesca o mediante leyes y decretos específicos (Decreto 152/1991; Decreto 61/1995; Decreto 145/1995; Ley 16/1998; Ley 22/2002; Ley 11/2008; Decreto 86/2004; Decreto 8/2014).

En Galicia hay 63 cofradías con un total de 12.734 miembros con diferentes niveles de dedicación (Federación Galega de CP, 2018). La mayoría tienen un tamaño similar a la pequeña empresa, aunque existe bastante diversidad en número de miembros y volumen de actividad. Estas organizaciones realizan su actividad sobre recursos marinos costeros, generalmente combinando pesca a pequeña escala, marisqueo y, en algunos casos, actividades acuícolas. Pueden ser miembros de las cofradías cualquier empresario o trabajador perteneciente a estos sectores -incluida cualquier modalidad pesquera-, por ejemplo, pescadores, mariscadores, armadores y propietarios de parques de cultivo entre otros. Entre las funciones que realizan, la normativa llega a atribuir a las cofradías funciones propias de la Administración Pública (Decreto 8/2014; García-Lorenzo et al, 2019b).

Este carácter público de las cofradías de pescadores ha sido el principal objeto de análisis de los estudios previos sobre ellas, centrados tradicionalmente en la perspectiva histórica e institucional. Con base a estos estudios, analizando su evolución y aplicando los marcos de análisis de la Nueva Economía Institucional (Mc Ginnis y Ostrom, 2014; Jentoft y Chuenpagdee, 2015), 
se llega a situarlas como actores de la pesca a pequeña escala, caracterizando su actividad, sistemas de recurso y estrategias (García-Lorenzo et al, 2019a).

Paralelamente a sus funciones públicas, las cofradías realizan actividades empresariales (Astorkiza et al, 2018). Estas asociaciones llevan a cabo actividades de organización, producción y comercialización del sector pesquero, marisquero y de acuicultura, las cuales son gestionadas por sus miembros dentro del marco normativo establecido (García-Lorenzo et al, 2019b). La capacidad de acción de las cofradías se muestra mayor en los niveles institucionales de acción colectiva y operacional, siendo el carácter autogestionario especialmente importante en este último nivel (Fernández-Vidal y Muiño, 2014; Alló y Loureiro, 2017; Ballesteros, 2018). Relacionado con esta autogestión, las leyes de Economía Social de España y Galicia incluyen a las cofradías de pescadores en su ámbito de aplicación (Cervera, 2010; Ley 5/2011; Ley 6/2016).

Su origen como agrupación privada, la posterior intervención de la administración, las funciones que realizan y su inclusión como entidades de Economía Social, hacen que la cofradía de pescadores se encuentren entre el ámbito privado y público. Este doble carácter, donde no siempre es posible separar ambos aspectos, influye en su estructura y las sitúa como entidades híbridas (Williamson, 2007; Mc Ginnis y 0strom, 2014: Jentoft y Chuenpagdee, 2015). Así, las cofradías son entidades asociativas con características y naturaleza público-privada, donde los miembros comparten y se benefician del uso y la explotación de los recursos marinos (García-Lorenzo et al, 2019a, 2019b). Este papel dentro del sector pesquero les otorga la posibilidad de contribuir al desarrollo sostenible, principalmente a nivel local.

\section{Influencia de las cofradías de pescadores de Galicia en el desarrollo sostenible}

En este apartado se analiza la influencia de las cofradías de pescadores de Galicia en dos Objetivos de Desarrollo Sostenible de la Resolución A/RES/70/1 de la Agenda 2030: el objetivo 14 de Vida Submarina y el 10 de Reducción de las Desigualdades. En concreto, la atención se centra en la protección de los recursos marinos costeros y de aguas interiores, en la equidad en el acceso a bienes comunes y en el reparto de rentas.

El apartado parte del estudio previo de las cofradías de pescadores, realizado desde el año 2015, y se divide en dos epígrafes, presentados como los principales puntos de unión con el desarrollo sostenible. Específicamente, se basa en el análisis teórico institucional sobre los procesos adaptativos de las cofradías (García-Lorenzo et al, 2019a) y en dos casos de estudio. El primero se centra en la participación de los miembros en las cofradías, desde la perspectiva de la Economía Social y la Economía de la Participación (García-Lorenzo et al, 2019b) y 
el segundo en el análisis de las interacciones de gobernanza en perspectiva institucionalista (García-Lorenzo y Varela-Lafuente, 2019).

Con estas bases, el estudio muestra una visión conjunta de las cofradías de pescadores de Galicia como entidades que pertenecen simultáneamente a dos ámbitos que, ya de forma individual, están bien posicionados para contribuir al desarrollo sostenible: la pesca a pequeña escala (WorldFish et al, 2018; FA0, 2020; Said et al, 2020) y la Economía Social (Bastida et al, 2020; Castro-Núñez et al, 2020; Chávez-Ávila y Gallego-Bono, 2020). El análisis integrado de esta doble posición busca profundizar en la capacidad que tienen las cofradías de pescadores de Galicia de contribuir a la consecución del ODS 10 y el ODS 14.

\subsection{Las cofradías dentro del sistema de cogestión de recursos marinos}

En la actualidad, la mayoría de los recursos marinos son recursos de uso común y no es posible ostentar su propiedad plena. Como consecuencia, el derecho a explotar el recurso no viene dado por su propiedad, sino por la propiedad de determinados instrumentos de gestión pesquera (TAC, cuotas, licencias, etc.). Estos instrumentos permiten la explotación durante un determinado período temporal y son, habitualmente, gestionados por las administraciones competentes. En la pesca, la Unión Europea establece cada año una cantidad máxima que puede ser capturada, calculada a través de estudios bioeconómicos y evoluciones históricas, la cual se reparte entre los Estados Miembros. A su vez, éstos determinan el reparto entre las diferentes regiones, como las Comunidades Autónomas, e incluso el reparto entre las propias embarcaciones pesqueras (González, 2006).

Más allá de la complejidad del sistema, esta organización no sigue un enfoque exclusivamente top down, sino que se crea un sistema de cogestión para la gobernanza de los recursos pesqueros. La UE y los Estados Miembros, incluso las organizaciones regionales de forma indirecta, pueden participar en las decisiones de repartición de los recursos, su gestión, control y vigilancia.

El caso del marisqueo es diferente, pues son recursos sedentarios localizados principalmente en aguas interiores, donde la Comunidad Autónoma de Galicia ostenta las competencias. Es más, dado el especial interés de la comunidad, el Tribunal Constitucional le atribuyó la competencia del marisqueo, independientemente de la zona interior o exterior de faena, dando prevalencia a la actividad frente al lugar (Barrio, 1998; Ballesteros, 2018). De esta forma, toda actividad sobre los recursos marisqueros, y parte de la pesca a pequeña escala realizada en aguas interiores, dependen de la regulación de la comunidad autónoma (Garza et al, 1996; Caballero et al, 2008; García-Lorenzo et al, 2019a). En consecuencia, el sistema de gobernanza de estos recursos es regional, y la cogestión entre las administraciones y las organizaciones o personas que ostenten el derecho de explotación, como pueden ser las cofradías, es más próxima.

En la pesca a pequeña escala y el marisqueo de Galicia, el sistema de gobernanza está conformado por las administraciones y las cofradías, pero no de manera exclusiva. El asociacionis- 
mo pesquero de la región cuenta con una amplia diversidad de organizaciones, algunas de las cuales también pertenecen a la Economía Social. El desarrollo histórico del sector ha generado agrupaciones de armadores tanto de pesca costera como de altura y gran altura (asociaciones y cooperativas de armadores; cooperativas del mar; clústeres de pesca), y otros grupos con distintos fines, más o menos estables. Para adaptarse a la normativa europea, también se constituyeron "organizaciones de productores", normalmente configuradas a partir de las asociaciones existentes (Cabaleiro et al, 2004; Caballero et al, 2008, 2014; Allo y Loureiro, 2017). Aunque no todas las experiencias han tenido éxito, estas asociaciones participan en la gestión pesquera de Galicia (García-Lorenzo et al, 2019b).

\section{Cuadro 1. Las cofradías de Galicia dentro del sistema de cogestión de recursos marinos}

\section{SISTEMA DE RECURSOS NATURALES}

Los sistemas de recursos sobre los que actúan son recursos costeros y de aguas interiores,

(Móviles -Caladero Nacional, Pesca en aguas interiores, Reservas Marinas- y Sedentarios -Marisqueo, Reservas Marinas-)

de los cuales se obtienen unidades de recurso como pescado, marisco y algas.

(Merluza, Sardina, Jurel, Caballa, Bonito del Norte, Pulpo, Almejas, Berberecho, Mejillón, Percebe, Erizo, Algas, etc.)

\section{SISTEMA DE GOBERNANZA}

El sistema de gestión de los recursos marinos es un sistema de cogestión, donde participan diferentes entidades

(Unión Europea, Estado Español, Comunidad Autónoma -Xunta de Galicia-, Cofradías de pescadores, Otras asociaciones de pescadores)

y se aplican instrumentos para la explotación sostenible de los recursos.

(TAC's y cuotas; Licencias y Control de outputs; Permisos de explotación; Concesiones, etc)

\section{COFRADÍAS DE PESCADORES}

Como actores y usuarios de estos sistemas, en Galicia hay 63 Cofradías de Pescadores con 12.734 miembros, principalmente armadores, pescadores y mariscadores.

Estas asociaciones desarrollan actividades de cogestión y explotación de los recursos, las cuales son auto-organizadas por sus miembros a través de los órganos sociales.

(Organización del sector; Producción pesquera, marisquera o acuícola; Comercialización de productos pesqueros; Actividades complementarias; Información; Procesos de decisión; Vigilancia; Control; Relaciones Externas)

Su estructura interna, determinada por la evolución histórica y el seguimiento de los principios cooperativos, influye en la forma de realizar estas actividades y en su relación con el medio.

(Adhesión libre y voluntaria; Gestión democrática; Participación económica; Autonomía e independencia; Educación, formación e información; Cooperación entre cooperativas; Interés por la comunidad)

Fuente: Elaboración propia a partir de McGinnis y Ostrom (2014) y García-Lorenzo et al (2019a,b). 
En el Cuadro 1 se observa la situación de las cofradías de pescadores de Galicia como actores de la pesca a pequeña escala y el marisqueo. Las cofradías están dentro de un sistema de gobernanza basado en la cogestión compartida entre diferentes entidades y que se articula a través de instrumentos de gestión. La finalidad es la explotación y protección de un sistema de recursos, en este caso recursos costeros y de aguas interiores. La participación de las cofradías en este sistema es doble, por su naturaleza pública colaboran como actores en la organización y, a su vez, son los usuarios que realizan la explotación privada de los recursos.

Las características y la importante posición que ocupan las cofradías en este sistema de gobernanza y cogestión contribuye a la consecución de la meta 14.b, facilitar el acceso de los pescadores tradicionales a los recursos marinos. En relación este acceso a los recursos dentro de la Unión Europea, el sector de la pesca a pequeña escala todavía sufre determinadas injusticias distributivas, tiene una baja capacidad para para beneficiarse de las oportunidades pesqueras y precisa de un mayor reconocimiento en las políticas europeas. Por ello, se hace relevante una mejor definición de los instrumentos de gestión y de asignación en los sistemas de gobernanza de múltiples niveles (Said et al, 2020). En este contexto, la fortaleza de las organizaciones regionales y la participación directa de los pescadores en los procesos de toma de decisiones supone una ventaja para asegurar los derechos de acceso al recurso.

Pero el logro de la meta 14.b requiere un enfoque holístico que abarque varios ODS, incluidos aquellos sobre el crecimiento económico, las comunidades sostenibles, las instituciones sólidas y las alianzas (Said y Chuenpagdee, 2019). La vinculación es especialmente fuerte con los objetivos relacionados con los medios de subsistencia, ya la pesca a pequeña escala desempeña un papel importante en la erradicación del hambre, la inseguridad alimentaria, la desnutrición y la pobreza en todo el mundo (FAO, 2020). Es más, en el análisis de escenarios futuros se prevé un aumento en la demanda mundial de alimentos y se plantea que aquellos obtenidos del mar puedan cubrir esta necesidad. Así, se calcula que en determinados escenarios los alimentos procedentes del mar podrían aumentar entre 21 y 44 millones de toneladas para el año 2050, donde la maricultura (de peces y bivalvos) cobraría una gran relevancia. Sin embargo, la realización sostenible de estos potenciales de producción dependerá de diversos factores, entre los que se encuentran las reformas políticas y la innovación tecnológica (Costello et al, 2020).

En estas reformas políticas se destaca la relevancia de la conservación del recurso y la gestión sostenible de la actividad pesquera, incluyendo la definición de límites de explotación basados en datos científicos (Costello et al, 2020). Estos aspectos se encuentran reflejados en diferentes metas del objetivo de Vida Submarina: gestionar y proteger sosteniblemente los ecosistemas marinos y costeros para evitar efectos adversos importantes (meta 14.29; aplicar planes de gestión con fundamento científico a fin de restablecer las poblaciones de peces en el plazo más breve posible (meta 14.4); mejorar la conservación y el uso sostenible de los océanos y sus recursos aplicando el derecho internacional reflejado en la Convención de las Naciones Unidas sobre el Derecho del Mar (meta 14.c).

La propia actividad pesquera de pequeña escala contribuye a la consecución de estas metas, al ser considerada menos agresiva para el medio ambiente y los ecosistemas en comparación 
con otros sectores pesqueros (FA0, 2015). Mientras que las cofradías de pescadores de Galicia tienen atribuidas funciones específicas de gestión y protección de los recursos marinos con el fin de desarrollar su actividad pesquera y marisquera de forma sostenible. Por un lado, relacionado con su carácter público, deben velar por el cumplimiento de la normativa, vigilar determinadas zonas de dominio público y colaborar con la Administración en elaboración de estadísticas, labores de inspección y recolección de datos. Por otro lado, llevan a cabo funciones que se sitúan entre lo público y lo privado, como la organización y gestión de planes de producción, de capturas y de comercialización. La participación de las cofradías en los instrumentos de gestión de la actividad marisquera es destacable. Las cofradías colaboran desde la fase de elaboración, estableciendo cada año el Plan General de Explotación Marisquera y otros Planes de Gestión específicos, de forma conjunta con la Xunta de Galicia. Estos planes son herramientas para la explotación sostenible y conservación de los recursos marinos, donde se estipulan los métodos de producción, las cantidades y los propietarios del derecho de explotación (Decreto 8/2014; Orden del Plan general de explotación marisquera para el trienio 2021-2023; Pesca de Galicia, 2021a).

Vinculado a la meta 14.5 de conservación de al menos el $10 \%$ de las zonas costeras y marinas, los pescadores de Galicia y sus cofradías han sido los responsables de impulsar dos reservas marinas de interés pesquero, estando involucrados en las propuestas desde el diseño y el desarrollo. Las reservas de "Os Miñazos" en Lira y de "Ría de Cedeira" en Cedeira son sistemas de gestión y ordenación pesquera que incluyen la preservación de los recursos y los hábitats, para lo cual se establecen Planes de Gestión Integrales de forma anual (Pesca de Galicia, 2021b). Sin embargo, la gestión de estas reservas no ha estado exenta de discusión, destacándose diversos problemas en su proceso de consolidación como la falta de flexibilidad, la elevada burocracia o la necesidad de establecer planes financieros y socioeconómicos a corto y largo plazo (Fernández Vidal y Muíño, 2014). Entre otros factores, esto se debe a que el establecimiento de zonas protegidas puede tener importantes repercusiones para las comunidades locales, especialmente aquellas dependiente del recurso. Una focalización exclusiva en los beneficios ambientales puede suponer una falta de consideración de los intereses y derechos de los habitantes locales y usuarios del recurso, como se ha analizado con determinados recursos costeros (Song et al, 2021). En el caso europeo se percibe que los conflictos con otros sectores económicos de la economía local, así como con los imperativos de conservación, crean problemas para la pesca a pequeña escala. El despojo del espacio marino y costero para el desarrollo de la actividad pesquera tradicional presenta un serio desafío, especialmente países del sur de Europa como España (Pita et al, 2020). Así, los grupos de interés, los pescadores y sus organizaciones deben participan de forma efectiva en la definición y gestión de las áreas marinas protegidas.

Así, las cofradías sí participan en los procesos de toma de decisiones dentro el sistema de cogestión de los recursos marinos de Galicia (García-Lorenzo et al, 2019a). Dependiendo de la actividad, son parte activa del proceso de decisión, son consultadas o, como mínimo, tienen el derecho a solicitar información sobre cualquier materia pesquera (Decreto 8/2014). Sin embargo, la cogestión implica la colaboración continua con administraciones europeas, na- 
cionales y regionales, y este sistema no está exento de problemas. Una demanda habitual es que las formas de representación de las cofradías en las administraciones son muy adecuadas, pero poco efectivas. Es decir, existen múltiples canales para participar en la toma de decisiones y sus propuestas son escuchadas, sin embargo, no son aplicadas. La actitud por parte de las administraciones suele ser más propensa a la centralización que a la cogestión y esto puede desincentivar la participación. Así, los conflictos surgen de manera recurrente por temas como el reparto de cuotas, la propuesta de nuevas leyes, la regulación del furtivismo, las medidas de seguridad o las sanciones, donde las cofradías presionan a favor de sus intereses (García-Lorenzo y Varela-Lafuente, 2019). La cogestión parece mostrarse más eficaz en las actividades marisqueras, donde las administraciones competentes son más próximas, los recursos sedentarios, el territorio de explotación delimitado y existe una mejor definición de los términos de uso de los derechos pesqueros (García-Lorenzo et al, 2019a).

Cofradías de pescadores de otras comunidades autónomas también se han analizado desde el punto de vista de la sostenibilidad, mostrando las diversas capacidades de esta forma organizativa. Así, en las cofradías mediterráneas se observa que la adaptación de algunos aspectos del modelo de cofradías podría producir formas más eficientes de gestión colaborativa y lograr mejoras en la sostenibilidad de la pesca. La adaptación a las condiciones ambientales locales y el control efectivo sobre el acceso a los recursos pesqueros que ejercen contribuyen tanto a ajustar y a hacer más efectiva la normativa general como a informar sobre los resultados observados en sus propias prácticas de autocontrol. Estas cofradías también facilitan la integración del conocimiento pesquero en el sistema de gestión y dan legitimidad a este sistema (Herrera-Racionero et al, 2020). Del mismo modo, el estudio de las comunidades pesqueras locales de la Isla de Tenerife muestra que su integración en los procesos de toma de decisiones puede ser una forma de promover la pesca artesanal, preservar el ecosistema marino y fomentar el desarrollo socioeconómico de las cofradías tradicionales. De hecho, entre los problemas para conseguir la sostenibilidad de sus pesquerías locales se identifican la baja capacidad de autogestión de las cofradías de la isla, el individualismo de los pescadores y la existencia de pocas estrategias de cogestión (Corral y Romero Marinque de Lara, 2017). En este sentido, no todas las cofradías de España ocupan la misma posición de poder dentro del sistema de cogestión (Bavinck et al, 2015), pero ésta puede ser fortalecida aprovechando la experiencia del sector en su conjunto.

\subsection{La actividad auto-organizada de las cofradías y los principios cooperativos}

Hasta ahora se ha analizado qué funciones que tienen encomendadas las cofradías dentro del sistema de gobernanza, sin embargo, el cómo afrontan estas funciones también puede suponer una ventaja para el desarrollo sostenible. Las cofradías no dejan de ser un grupo de pescadores y mariscadores que se asocian voluntariamente para explotar un recurso de forma común. Como grupo, la estructura interna y funcionamiento de las cofradías están condicionados por 
dos características principales: (1) su origen tradicional y los procesos sufridos para adaptarse a las condiciones históricas; (2) su carácter privado auto-organizado y el cumplimiento de los principios cooperativos, que la han llevado hasta su actual definición como entidad de Economía Social.

La inclusión de las cofradías de pescadores dentro de la Economía Social las posiciona en un sector al que la propia Agenda 2030 otorga un papel importante en la consecución de los Objetivos de Desarrollo Sostenible (Chávez-Ávila y Gallego-Bono, 2020). En concreto, la Economía Social ha mostrado su capacidad de contribución a los ODS 5 de Igualdad de Género, ODS 8 de Trabajo Decente y Crecimiento Económico y ODS 10 de Reducción de las Desigualdades, tanto a nivel español (Castro-Núñez et al, 2020) como a nivel gallego (Bastida et al, 2020). Pero además de ser importante para el desarrollo de un enfoque participativo, inclusivo y equitativo, la Economía Social es considerada relevante para el medio ambiente, especialmente a través su anclaje territorial (Chávez-Ávila y Gallego-Bono, 2020).

Este anclaje también existe para el caso de las cofradías, ya los pescadores y mariscadores están ligados al territorio donde desarrollan su actividad productiva (Decreto 8/2014), percibiendo al recurso como un medio de subsistencia para su entorno y familia. Este hecho, junto con la larga historia asociativa de la que proviene la cofradía, hace que existan procesos de continuidad en la relación con el medio ambiente y que se produzca un aprovechamiento de la tradición en su explotación y conservación. Así, los miembros de las cofradías realizan las labores de producción pesquera y marisquera de modo colectivo, aplicando métodos de producción tradicionales. En la pesca, los armadores y pescadores cooperan al extraer el recurso con sus propios medios de producción, mientras que en el marisqueo la cooperación es mayor. Los mariscadores extraen el recurso de forma conjunta, pero también participan en otras labores colectivas establecidas en los Planes de Gestión, como la siembra, limpieza y vigilancia (Orden del Plan general de explotación marisquera para el trienio 2021-2023; Pesca de Galicia, 2021a; García-Lorenzo et al, 2019b).

Los procesos adaptativos hicieron que la cooperación necesaria en las tareas productivas se extendiese también a la comercialización, con la finalidad de no competir en los mercados (González-Arce, 2011; García-Lorenzo et al, 2019a). Así, en la actualidad, las cofradías venden de manera conjunta los productos obtenidos de la explotación de recursos a través de las lonjas -mercado para la exhibición y venta de productos frescos- (Ley 3/2001). En Galicia, alrededor del 75\% de las cofradías gestionan su propia lonja y pueden decidir sobre la estrategia operacional, como las tasas a pagar, las horas de apertura o las formas de venta. Esta forma colectiva de comercialización es el principal ingreso económico para muchas cofradías (García-Lorenzo et al, 2019b).

La evolución en la forma organizativa de la cofradía ha hecho que los pescadores y mariscadores auto-organicen estas labores de producción y la comercialización a través de sus órganos sociales, cumpliéndose con el principio de gestión democrática. Estos órganos representan a la cofradía frente a la administración, se eligen democráticamente y están formados por los propios miembros. A su vez, el sistema de toma de decisiones dentro de ellos se realiza de forma democrática, cada representante tiene derecho a un voto. Los pescadores gestio- 
nan su actividad y tienen vías directas para informarse y comunicar sus problemas, como el tablón de anuncios, el correo electrónico o medios informales. Este sistema es más complejo que una persona un voto, debido a la variedad de miembros que conviven en la cofradía, lo cual representa algunas desventajas. Puede darse el caso de la infrarrepresentación de algún colectivo y el número de mujeres representantes es casi exclusivo del sector marisquero (Decreto 8/2014; García-Lorenzo et al, 2019b). Pese a ello, la participación de los miembros en los órganos sociales, y la participación de estos en el sistema de gobernanza pesquero, provee un canal de información y comunicación entre los productores y las administraciones (aunque no siempre efectivo). En general, existe una fuerte identificación de los gestores con sus cofradías, los miembros están muy satisfechos con la convivencia dentro de la organización y se perciben muy pocos conflictos internos (solo puntualmente) por motivos de edad, sexo, ideología o sector de producción (García-Lorenzo y Varela-Lafuente, 2019).

La actividad auto-organizada de las cofradías tiene repercusiones en la protección de los recursos y en la reducción de las desigualdades. Relacionado con las metas 14.2 y 14.c anteriormente mencionadas, la vinculación de los pescadores con el medio y la normativa favorecen las actividades menos intensivas y agresivas con el medio marino. Los métodos tradicionales de producción de la pesca a pequeña escala y el marisqueo, las labores de vigilancia y las restricciones en la explotación son algunas de las estrategias de protección del medio. Aun así, se aprecian dos características relevantes del comportamiento de los pescadores que pueden suponer una desventaja: (1) una actitud conservadora frente a la gestión pesquera, que protege el recurso, pero puede desfavorecer la innovación y acciones de mejora; (2) y una visión cortoplacista en busca de intereses individuales, que choca con el objetivo a largo plazo de la sostenibilidad (García-Lorenzo et al, 2019a). A su vez, que sean los propios pescadores y mariscadores quienes participen en la cogestión de los recursos es una fuente importante de conocimiento para estudiar los problemas de la sostenibilidad. Por ejemplo, se aprecia que los pescadores perciben el declive de los stocks de peces, la pérdida de hábitats y la contaminación de los mares como los problemas medioambientales más relevantes, relegando otros como la urbanización costera, las aguas residuales o la erosión costera (Stobberup et al, 2017). El conocimiento de estas actitudes puede ser relevante para una adecuada planificación de las estrategias futuras en gestión del litoral.

En relación a la reducción de las desigualdades dentro de los países, la capacidad de contribución de las cofradías se centra en lograr progresivamente y mantener el crecimiento de los ingresos del $40 \%$ más pobre de la población (meta 10.1) y garantizar la igualdad de oportunidades y reducir la desigualdad de resultados (meta 10.3). En primer lugar, las cofradías llevan a cabo la producción y la comercialización de productos pesqueros como actividad empresarial y privada que cumple con el principio de participación económica. Al igual que en las cooperativas, no es posible pertenecer a la cofradía si no se realiza una actividad pesquera, marisquera o acuícola, es decir, si no se participa en la actividad real de la organización. De esta forma, los resultados de la actividad empresarial se aplican en función del trabajo o servicio aportado por los pescadores, no por la aportación de capital a la entidad. La renta residual se traslada a los miembros a través de la ventaja en precios, y el análisis muestra que la cofradía 
ofrece los mejores precios posibles a sus miembros, llegando a ser los resultados económicos muy reducidos o nulos (García-Lorenzo et al, 2019b). Este cumplimiento del principio de participación económica favorece el reparto equitativo de rentas sobre la acumulación de capital (Cabaleiro, 2004) aunque como contrapartida se pueda dificultar el uso de nuevas estrategias y las grandes inversiones tecnológicas.

En segundo lugar, muchas de las cofradías desarrollan su actividad en un entorno rural costero (principalmente aquellas de la costa cantábrica y la costa da morte), siendo estos territorios considerados como zona intermedias de baja población o incluso zonas poco pobladas (IGE, 2021). Realizar una actividad empresarial en un entorno local y rural tiene un potencial de redistribución de la actividad económica que conlleva ventajas para el desarrollo endógeno y sostenible de la región como: (1) la fijación de población y menor necesidad de flujos migratorios; (2) el establecimiento de canales para aplicar las políticas de desarrollo rural; (3) y la generación de valor añadido a la región, mediante el cual la riqueza creada revierte en los actores que la han originado (Cabaleiro, 2004).

Además de las actividades de producción y de comercialización, las cofradías realizan otras actividades complementarias que se relacionan con los principios cooperativos de educación, formación e información e interés por la comunidad. Estas actividades se centran en la prestación de servicios y pueden ser: formativas -cursos de marisqueo, de títulos de pesca, gestión cooperativa; medidas de seguridad y sanidad o protección de los recursos-; administrativas -renovación de permisos, asesoría o impuestos-; turísticas o de conservación del patrimonio cultural y ambiental -gestión de museos, lonjas turísticas, turismo marinero, fiestas patronales o colaboración en clubes deportivos- (García-Lorenzo et al, 2019b, García-Lorenzo y Varela-Lafuente, 2019). Del mismo modo, la cooperación entre cooperativas también está presente en el sector, habiendo una Federación Gallega de Cofradías de Pescadores y tres Federaciones Provinciales. Estas organizaciones facilitan la representación de sus intereses como interlocutores reconocidos y organizan múltiples actividades. Igualmente, las cofradías colaboran de manera bilateral entre ellas, normalmente con entidades próximas geográficamente (Federación Galega de CP, 2018; Federación Provincial de CP de Lugo, 2021).

Al realizar su actividad sobre recursos comunes, hay una característica de las empresas de Economía Social que influye en la equidad en el acceso a bienes. Las cofradías cumplen el principio de adhesión libre y voluntaria, de forma que cualquier persona que tenga los requisitos necesarios para realizar la actividad pesquera y marisquera puede ser miembro. No es posible excluir a nadie de la explotación de un recurso común por motivos subjetivos de una cofradía, solamente en función de estos requisitos legales establecidos para evitar la sobreexplotación y asegurar la correcta realización de la actividad. Cualquier persona que los posea puede entrar a forma parte la cofradía y, consecuentemente, del proceso de toma de decisiones interno y externo (García-Lorenzo et al, 2019b). Esto se ve reflejado en el análisis financiero de las cofradías, donde el resultado del ejercicio, cuando es positivo, revierte a la cofradía y permanece como fondos propios de la entidad. Los miembros no tienen derecho a una participación en estos beneficios ni a una compensación económica a su salida de la cofradía, como pueden ser el retorno cooperativo o la aportación al capital social de las sociedades cooperativas 
(Cabaleiro, 2004). Consecuentemente, parte de los beneficios de la explotación de un recurso común se comparten con la sociedad local. Esta característica se puede relacionar tanto con la consecución del acceso de los pescadores artesanales a los recursos marinos como con la redistribución de resultados.

Las cofradías, administraciones y principalmente las mariscadoras, también han jugado un papel importante en el acceso de la mujer al mercado laboral. La pesca a pequeña escala es una actividad realizada tradicionalmente por núcleos familiares, donde la mujer siempre ha estado presente. En el caso de Galicia, las mujeres se concentraban en la fabricación de redes, las fábricas de procesado de productos pesqueros y en el marisqueo a pie. Hasta la década de los sesenta el marisqueo era considerado una actividad de autoconsumo y subsistencia y, tras muchos esfuerzos e intentos, en la actualidad es una actividad profesionalizada realizada mayormente por mujeres (Marugán, 2004). Las mariscadoras cuentan con agrupaciones propias, representación dentro los órganos sociales de las cofradías e importantes poderes de decisión en el nivel operativo, llegando a ser reconocido a nivel internacional su proceso de profesionalización (Frangoudes et al, 2008; Alonso-Población y Siar, 2018). De esta forma, las cofradías contribuyen a potenciar y promover la inclusión social, económica y política de todas las personas (meta 10.2) y se influencian determinados aspectos del ODS 5 de Igualdad de Género. En concreto, aquellos centrados en asegurar la igualdad de oportunidades de liderazgo (meta 5.5) y otorgar a las mujeres igualdad de derechos a los recursos económicos, así como acceso a la propiedad y al control de la tierra y los recursos naturales (meta 5.a).

Hay que destacar que la presencia de la mujer en las cofradías sigue estando muy relacionada al marisqueo, pero actualmente también existen mujeres armadoras o pescadoras. Otro factor positivo es que las cofradías no perciben conflictos internos destacables por motivos de género, solo destacando problemas específicos en situaciones concretas (García-Lorenzo y Varela-Lafuente, 2019). Las cofradías pueden ser una experiencia útil para estudiar la participación real de la mujer en el sector pesquero, la cual todavía es excluida de muchos análisis y para la que aún existe una falta de datos (Kleiber et al, 2015). Además, actualmente se destaca la necesidad de adoptar una perspectiva de género junto con la recopilación de datos, a fin de permitir el estudio de las complejas interacciones de poder y las relaciones entre mujeres y hombres en la pesca y la acuicultura (FA0, 2020).

Por último, al realizar una actividad empresarial cumpliendo con la filosofía de la Economía Social y estar sometida a un fuerte control derivado de su carácter público-privado, la actuación de las cofradías también genera sinergias con el ODS 8 de Trabajo Decente y Crecimiento Económico. Su desarrollo e implantación favorece la creación de puestos de trabajo decentes y de pequeñas y medianas empresas (ya que en su consideración de empresa la mayoría de las cofradías se enmarcarían como pequeña empresa, solo llegando en algunos casos al tamaño mediano (García-Lorenzo et al, 2019b)). Del mismo, la fuerte posición que ocupan las cofradías en el sector pesquero, las obligaciones de rendición de cuentas a las que se ve sometida por su carácter público (Decreto 8/2014) y la estructura democrática y participativa que tienen, favorece la consecución de instituciones eficaces y transparentes, del ODS 16 de Paz, Justicia e Instituciones. 


\title{
Cuadro 2. Capacidad de contribución de las cofradías de pescadores de Galicia a los Objetivos de Desarrollo Sostenible 10 y 14
}

\author{
ODS 14, DE VIDA SUBMARINA \\ Conservar y utilizar sosteniblemente los océanos, los mares y los recursos marinos
}

Facilitar el acceso de los pescadores artesanales a los recursos marinos y los mercados (meta 14.b) $\rightarrow$ Involucrar en la cogestión de recursos pesqueros a las comunidades locales; Adhesión libre y voluntaria a una organización que cogestiona recursos naturales comunes; Capacidad de organización de una multiplicidad de actores; Mejorar la definición del grupo (quién puede ser miembro) y de sus derechos de explotación frente a terceros; Favorecer la participación de los pescadores en la toma de decisiones y legitimización de estas decisiones gracias a la fuerte posición de las cofradías; Desarrollar de forma colectiva de la venta de productos pesqueros.

Gestionar sosteniblemente los ecosistemas marinos y costeros (meta 14.2), aplicar planes de gestión con fundamento científico (meta 14.4) y mejorar la conservación y el uso sostenible de los océanos y sus recursos (meta 14.c) $\rightarrow$ Percepción del recurso como medio de subsistencia y como valor cultural; Proporcionar conocimiento local sobre temas ambientales; Emplear métodos tradicionales de producción de la pesca a pequeña escala y el marisqueo; Incorporar asistencia técnica y personal biólogo en las cofradías; Colaborar directamente con las AAAPP en la elaboración de estadísticas, labores de inspección y recolección de datos técnicos; Desarrollar funciones de protección de los recursos, como las labores de control y vigilancia y la co-elaboración y seguimiento de planes de gestión (destacando el Plan General y los Planes de Gestión específicos para la explotación marisquera).

Conservar al menos el $10 \%$ de las zonas costeras y marinas (meta 14.5$) \rightarrow$ Los pescadores locales y sus cofradías sido los responsables de impulsar dos reservas marinas de interés pesquero, estando involucrados en las propuestas desde el diseño y el desarrollo. Las zonas conservación deben estar bien definidas y la participación de los pescadores debe ser efectiva, ya que están zonas pueden generar problemas en la definición de determinados derechos.

Sinergias con el ODS 1 de Fin de la Pobreza y ODS 2 de Hambre Cero y seguridad alimentaria $\rightarrow$ La pesca artesanal y la acuicultura juegan un papel clave en la erradicación del hambre, la inseguridad alimentaria, la desnutrición y la pobreza, siendo especialmente importante garantizar los derechos de pesca de las comunidades locales para conseguir estos objetivos. El potencial de crecimiento de la pesca y acuicultura para satisfacer la demanda de alimentos futura es especialmente destacable en aquellas actividades realizadas en aguas interiores o costeras (como la maricultura), donde las cofradías de pescadores tienen mayores competencias y capacidad de influencia.

ODS 10, DE REDUCCIÓN DE LAS DESIGUALDADES

Reducir la desigualdad en y entre los países

Lograr progresivamente y mantener el crecimiento de los ingresos del $40 \%$ más pobre de la población a una tasa superior a la media nacional (meta 10.1) y garantizar la igualdad de oportunidades y reducir la desigualdad de resultados (meta 10.3) $\rightarrow$ Reparto equitativo de rentas debido a que la obtención de los beneficios de la actividad económica en función del trabajo o servicio prestado, no del capital aportado; Reducción de costes de transacción de una actividad económica tradicional al realizarla de manera colectiva; Generación de empleo rural y valor añadido al territorio, fijación poblacional; Realizar otras actividades que contribuyen a la sociedad local; Fomentar la cooperación entre las cofradías y sus territorios a través de las federaciones provinciales y autonómicas.

Potenciar y promover la inclusión social, económica y política de todas las personas (meta 10.2) y sinergias con el ODS 5, de Igualdad de Género, especialmente con la igualdad de liderazgo (meta 5.5) y la igualdad de derechos a los recursos económicos, así como acceso a la propiedad y (...) y al control de los recursos naturales (meta 5.a) $\rightarrow$ Profesionalización del sector del marisqueo a pie; Reconocimiento, definición y gestión de los derechos de explotación de recursos marisqueros; Participación de las mujeres en sus propias agrupaciones de mariscadoras y en los órganos de gestión de la cofradía; Posibilidad de aplicar la perspectiva de género en el estudio del sector pesquero.

Sinergias con el ODS 8 de Trabajo Decente y Crecimiento Económico y el ODS 16 de Paz, Justicia e Instituciones sólidas $\rightarrow$ En su consideración de empresa, la mayoría de las cofradías tienen un tamaño similar a una pequeña empresa, en algunos casos mediana; Sistema democrático para la elección de sus órganos sociales; Legitimización de una institución público-privada, que tiene una base comunitaria y sigue principios de transparencia para la rendición de cuentas.

Fuente: Elaboración propia a partir de la Resolución A/RES/70/1 de la Agenda 2030. 
En consecuencia, el análisis integrado de la doble posición de las cofradías de pescadores de Galicia, como entidades de Economía Social que desarrollan su actividad en el sector de la pesca a pequeña escala y el marisqueo, permite profundizar en su capacidad de contribución al ODS 10 y al ODS 14 (Cuadro 2).

\section{Conclusiones}

Las cofradías de pescadores de Galicia juegan un papel importante en la gestión y explotación de los recursos marinos. Esta posición les permite influir en algunos aspectos identificados en la Agenda 2030 para el Desarrollo Sostenible, como la protección de los recursos y la equidad en el reparto de ingresos y en el acceso a bienes comunes. Las principales causas que sustentan esta influencia son su situación como actores y usuarios dentro del sistema de cogestión pesquera y marisquera y su estructura organizativa de naturaleza público-privada. Esta estructura nace de la adaptación a las condiciones institucionales históricas y del carácter auto-organizado, y ha llevado a las cofradías a enmarcarse como entidades de Economía Social que cumplen los principios cooperativos.

Las cofradías comparten la gestión de los recursos costeros y de aguas interiores con las administraciones públicas y otras entidades del sector, protegiéndolos de manera conjunta y contribuyendo al objetivo de Vida Submarina y sus metas 14.2, 14.4, 14.b y 14.c. En concreto, ellas colaboran en la organización del sector, en las labores de control y vigilancia, y en la gestión de planes de producción y comercialización sostenibles. Para cumplir con estos cometidos, las cofradías participan activamente en los procesos de tomas de decisiones de la cogestión pesquera, aunque esta participación no siempre es efectiva. Las administraciones tienden a tener una actitud centralizada y surgen múltiples conflictos. En este sentido, las políticas públicas deberían favorecer una participación más eficaz de las cofradías. En entornos locales, la inclusión de las demandas de los usuarios en los procesos de decisión, así como la concesión de una mayor capacidad de auto-organización, mejora la cogestión del recurso. Esta situación se muestra en el caso del marisqueo, donde las administraciones competentes son regionales y están próximas a las cofradías.

La vinculación al territorio de estas organizaciones y la forma colectiva de explotación también fomenta la percepción del recurso como un medio de subsistencia y aporta resiliencia al sector. Se mantienen los métodos tradicionales de producción, haciendo la actividad menos intensiva y agresiva con el medio marino, y se consigue un conocimiento más cercano y real del recurso, obteniendo información útil para la gestión pesquera. A pesar de esto, se aprecian actitudes conservadoras y cortoplacistas entre los pescadores, derivadas de la confluencia de intereses, que pueden ir en contra del desarrollo sostenible. Las futuras políticas deberían encaminarse hacia el fomento de la sostenibilidad a largo plazo y desincentivar estas actitudes cortoplacistas. Mejorar la regulación sobre sanciones y proporcionar una línea de continuidad en las políticas, donde los pescadores y mariscadores sientan seguros sus derechos sobre el recurso, fomentaría su visión a largo plazo. 
Por otro lado, las cofradías llevan a cabo su actividad empresarial en un entorno local y rural, impulsando el desarrollo endógeno de la región a través de la fijación de población y la generación de valor añadido. Que esta actividad se realice de manera colectiva, democrática y cumpliendo con el principio de participación económica contribuye a la consecución del objetivo de Reducción de Desigualdades y sus metas 10.1, 10.2 y 10.3. Al percibir las rentas en función del trabajo y no de la aportación de capital, se favorece un reparto más equitativo de los beneficios entre los pescadores y mariscadores, incluidas las mujeres. Esta es una característica distintiva de las empresas de Economía Social que, en el caso de las cofradías, tiene un segundo impacto positivo. La explotación cooperativa del ámbito marino reduce las desigualdades de acceso a un recurso de propiedad común y revierte parte de sus beneficios en la sociedad en su conjunto.

\section{Agradecimientos}

La autora agradece el apoyo financiero de FEDER y Xunta de Galicia (ED431C2018/48 y ED431E2018/07), y del Ministerio de Economía y Competividad de España (RTI2018099225-B-100).

\section{Bibliografía y referencias normativas}

ALLÓ, M. \& LOUREIRO, M.L. (2017): "The role of social norms on conservation programmes in shellfish fisheries”, Marine Policy, 84, 134-141. D0I: 10.1016/j.marpol.2017.07.008.

ALONSO-POBLACIÓN E. \& SIAR S.V. (2018): “Women's participation and leadership in fisherfolk organizations and collective action in fisheries: A review of evidence on enablers, drivers and barriers", FAO Fisheries and Aquaculture Circular No. 1159, Rome, FA0, ISBN 978-92-5130245-3.

ARNASON, R. (1989): "Minimum Information Management with Help of Catch Quotas", In P. Neher, R. Arnason y N. Mollet (eds.), Right Based Fishing. Netherlands: Kluwer Academic Publishers: 215-240.

ARNASON, R. (1994): "Theoretical and practical fishery management”. In E.A. Loayza (ed.), Managing Fishery Resources, Washington D.C: World Bank Discussion Papers, Fishery Series: 3-10.

ASTORKIZA, K. \& DEL VALLE, I. (2018): "An economic analysis of private side of fishermen's cofradías' activity on the Cantabrian Sea", Marine Policy, 90, 152-159.

DOI: 10.1016/j.marpol.2017.12.018. 
BALLESTEROS, M.A. (2018): “Gobernanza policéntrica en sistemas socioecológicos complejos: la gestión de la pesquería del pulpo (Octopus vulgaris) en Galicia", Tesis Doctoral, Universidad de Vigo.

BARRIO, G. (1998): Régimen jurídico de la pesca marítima, Ed. Marcial Pons, Madrid.

BASTIDA, M., VAQUERO GARCÍA, A., CANCELO MÁRQUEZ, M. \& OLIVEIRA BLANCO A. (2020): "Fostering the Sustainable Development Goals from an Ecosystem Conducive to the SE: The Galician's Case", Sustainability, 12(2), 500. DOI: 10.3390/su12020500.

BAVINCK, M., JENTOFT S., PASCUAL-FERNÁNDEZ, J.J. \& MARCINIAK, B. (2015): "Interactive coastal governance: The role of pre-modern fisher organizations in improving governability", Ocean \& Coastal Management, 117, 52-60. D0I: 10.1016/j.ocecoaman.2015.05.012.

BJORNDAL, T. \& MUNRO, G. (2012): The Economics and Management of World Fisheries, Oxford University Press, ISBN 978-0- 19-957675.

CABALEIRO-CASAL, M.J. (2004): "La intercooperación de las sociedades cooperativas. Su aplicación en el desarrollo rural integral de la Comunidad Autónoma de Galicia", Tesis Doctoral, Consellería de Asuntos Sociais, Emprego e Relacións Laborais, Xunta de Galicia.

CABALEIRO-CASAL, M.J., ANDRÉ-FERNÁNDEZ, R. \& GÓMEZ-CORTÉS, S. (2004): “Las sociedades cooperativas del mar: impedimentos para su implantación en Galicia", REVESCO, Revista de Estudios Cooperativos, 84, 7-35.

CABALLERO-MIGUEZ, G., GARZA-GIL, M.D. \& VARELA-LAFUENTE, M.M. (2008): "Institutions and Management of Fishing Resources: The governance of the Galician model", Ocean \& Coastal Management, 51, 8-9, 625-631. DOI: 10.1016/j.ocecoaman.2008.06.003.

CABALLERO-MIGUEZ, G., VARELA-LAFUENTE, M.M. \& GARZA-GIL, M.D. (2014): "Institutional change, fishing rights and governance mechanisms. The dynamics of the Spanish 300 fleet on the Grand Sole fishing grounds", Marine Policy, 44, 465-472.

D0I: 10.1016/j.marpol.2013.10.015.

CASTRO NÚÑEZ, R.S., BANDEIRA R. \& SANTERO-SÁNCHEZ R. (2020): “Social Economy, Gender Equality at Work and the 2030 Agenda: Theory and Evidence from Spain", Sustainability, 12, 5192. DOI: $10.3390 /$ su12125192.

CERVERA, A. (2010): "Percepción cooperativa de las cofradías de pescadores: un estudio empírico", REVESCO, Revista de Estudios Cooperativos, 102, 3, 7-32.

CHAVES-ÁVILA R. \& GALLEGO-BONO J.R. (2020): "Transformative Policies for the Social and Solidarity Economy: The New Generation of Public Policies Fostering the Social Economy in Order to Achieve Sustainable Development Goals. The European and Spanish Cases", Sustainability, 12, 4059. D0I: 10.3390/su12104059. 
CLARK, C.W. \& MUNRO, G.R. (1975): "The economics of fishing and the modern capital theory: A simplified approach", Journal of Environmental Economics and Management, 2, 2, 92-106.

COMISIÓN EUROPEA (2016): "Small Scale Coast Fleet in the EU”. https://ec.europa.eu/fisheries/sites/fisheries/files/docs/publications/2016-small-scale-coastal-fleet_en.pdf (acceso el 27.08.18).

CORRAL, S. \& ROMERO MARINQUE DE LARA, D. (2017); "Participatory artisanal fisheries management in islands: Application to the Canary Islands (Spain)", Marine Policy, 81:45-52. DOI: $10.1016 /$ j.marpol.2017.03.011.

COSTELLO, C., CAO, L. \& GELCICH, S. et al. (2020): “The future of food from the sea”, Nature 588, 95-100. DOI: 10.1038/s41586-020-2616-y.

FAO (2015): "Voluntary Guidelines for Securing Sustainable Small-Scale Fisheries in the Context of Food Security and Poverty Eradication", Rome, Italy.

FAO (2020): "The state of world fisheries and aquaculture 2020. Sustainability in action", Rome, Italy. D0I: 10.4060/ca9229en.

FEDERACIÓN GALEGA DE COFRADÍAS DE PESCADORES (2018): "Cofradías". http://confrariasgalicia.org/ (acceso el 27.08.18).

FEDERACIÓN PROVINCIAL DE COFRADÍAS DE PESCADORES DE LUGO (2021): “La federación”. http://cofradiaslugo.com/es/ (acceso el 11/03/2021).

FERNÁNDEZ-VIDAL, D. \& MUIÑO, R. (2014): "Fact or fiction? Assessing governance and co-management of Marine Reserves of Fishing Interest in Cedeira and Lira (NW Spain)", Marine Policy, 47,15-22. D0I: 10.1016/j.marpol.2014.01.016.

FRANGOUDES, K., MARUGÁN-PINTOS, B. \& PASCUAL-FERNÁNDEZ, J.J. (2008): “From open access to cogovernance and conservation: The case of women shellfish collectors in Galicia (Spain)", Marine Policy, 32(2): 223-232. D0I: 10.1016/j.marpol.2007.09.007.

GARCÍA-LORENZO, I., VARELA-LAFUENTE, M.M. \& GARZA-GIL, M.D. (2019a): "Adaptative processes in small-scale traditional fishermen's organisations. The case of the Cofradías in Galicia (NW Spain)", Marine Policy, 99, 382-390. D0I: 10.1016/j.marpol.2018.10.041.

GARCÍA-LORENZO, I., CABALEIRO-CASAL, M.J. \& VARELA-LAFUENTE, M.M. (2019b): “Fishermen's Associations of the small-scale fisheries: Study applied to the participation in Cofradías of Galicia (MW Spain)", Ocean \& Coastal Management, 178, 104841.

DOI: 10.1016/j.ocecoaman.2019.104841. 
GARCÍA-LORENZO, I. \& VARELA-LAFUENTE, M.M. (2019): "Interacciones de gobernanza en las pesquerías a pequeña escala de Galicia: Percepciones y dinámicas en las cofradías de pescadores", Estudios de Economía Aplicada, 37, 3, 42-59. D0I: 10.25115/eea.v37i3.2772.

GARZA-GIL, M.D., IGLESIAS-MALVIDO, C., SURÍS-REGUEIRO, J. \& VARELA-LAFUENTE, M.M. (1996): “The Spanish case regarding fishing regulation", Marine Policy, 20, 3, 249-259.

DOI: 10.1016/0308-597X(96)00007-3.

GARZA-GIL, M.D. \& VARELA-LAFUENTE, M.M (2005): "The profitability of the Spanish swordfish fleet in the North Atlantic", Marine Policy, 29, 6, 533-538.

DOI: https://doi.org/10.1016/j.marpol.2004.11.001.

GONZÁLEZ-ARCE, J.D. (2011): "Análisis comparativo de las cofradías de pescadores de Castilla (siglos XII-XV)", Historias, Instituciones, Documentos, 38, 141-217.

GONZÁLEZ-LAXE, F. (2006): "Transferability of fishing rights: The Spanish case", Marine Policy, 30, 379-388. DOI: https://doi.org/10.1016/j.marpol.2005.06.008.

GORDON, H.S. (1954): "Economic Theory of Common Property Resource: the Fishery", Journal of Political Economy, 62, 124-142.

HERRERA-RACIONERO P., LIZCANO E., MIRET-PASTOR L. \& MASCARRELL Y. (2019): "The Spanish Mediterranean Fishing Guilds (Cofradías): An example of collaborative management with key role in sustainable fisheries", Fisheries, 44, 4: 172-182. D0I: 10.1002/fsh.10224.

INSTITUTO GALEGO DE ESTATÍSTICA -IGE- (2021): "Mapa dos concellos de Galicia segundo o subgrao de urbanización (GU 2016)". https://www.ige.eu/estatico/estat.jsp?ruta=html/gl/ rural-urbano/MapaConcellosSubgrao2016.htm el (acceso 11/03/2021).

INTERNATIONAL ASSOCIATION FOR THE ECONOMICS OF PARTICIPATION (IAFEP) (2019): http://www.iafep.org/ (acceso el 24/01/2019).

JENTOFT, S. (2004): "Institutions in fisheries: what they are, what they do, and how they change?", Marine Policy, 28, 137-149. DOI: 10.1016/S0308-597X(03)00085-X.

JENTOFT, S. \& CHUENPAGDEE, R. (Eds.) (2015): Interactive governance for small-fisheries. Global Reflections, Centre for Maritime Research MARE, Springer Int. Pub. Switzerland.

KLEIBER, D., HARRIS, L.M. \& VICENT, A.C.J. (2015): “Gender and small-scale fisheries: a case for counting women and beyond", Fish and Fisheries, 16, 547-562. DOI: 10.1111/faf.12075.

LABARTA, U. (1979): “Galicia mariñeira. Historia económica e científica”, en VV.AA., Estudio y explotación del mar en Galicia, 11-72, Santiago de Compostela. 
LINKE, S. \& BRUCKMEIER, K. (2015): “Co-management in fisheries. Experiences and changing approaches in Europe", Ocean \& Coastal Management, 104, 170-181.

DOI: 10.1016/j.ocecoaman.2014.11.017.

MARUGÁN-PINTOS, B. (2004): E colleron ese tren... Profesionalización das mariscadoras galegas, Consellería de Pesca e Asuntos Marítimos, Xunta de Galicia, ISBN: 84-453-3927-3.

MC GINNIS, M.D. \& OSTROM, E. (2014): "Social-ecological system framework: initial changes and continuing challenges", Ecology and Society, 19, 2, 30. D0I: 10.5751/ES-06387-190230.

OSTROM, E. (1990): Governing the commons. The evolution of institutions for collective action, Cambridge University Press, Cambridge.

PESCA DE GALICIA, 2021a. "Planes de Gestión".

https://www.pescadegalicia.gal/PlanesExp/index.htm (acceso el 11/03/2021).

PESCA DE GALICIA (2021b): "Reservas mariñas”.

https://www.pescadegalicia.gal/gl/reservas-marinas (acceso el 11/03/2021).

PITA C., PASCUAL-FERNÁNDEZ J.J. \& BAVINK M. (2020): "Small-Scale Fisheries in Europe: Challenges and Opportunities", In: J.J. Pascual-Fernández, C. Pita \& M. Bavinck (Ed.), SmallScale Fisheries in Europe: Status, Resilience and Governance (pp. 581-600), MARE Publications Series, 23, Springer Cham. DOI: 10.1007/978-3-030-37371-9_28.

SAID, A. \& CHUENPAGDEE, R. (2019): "Aligning the sustainable development goals to the smallscale fisheries guidelines: A case for EU fisheries governance", Marine Policy, 107, 103599. D0I: 10.1016/j.marpol.2019.103599.

SAID, A. et al (2020): "Small-scale fisheries access to fishing opportunities in the European Union: Is the Common Fisheries Policy the right step to SDG14b?", Marine Policy, 118,104009. DOI: $10.1016 /$ j.marpol.2020.104009.

SCHAEFER, M.B. (1957): "Some considerations of the dynamics of population important to the management of the commercial marine fisheries", Journal of Fisheries Researchs Board of Canada, 14, 5, 669-681.

SCHLAGER, E. \& OSTROM, E. (1992): "Property-rights regimes and natural resources: a conceptual analysis", Land Economics, 68, 3, 249-262.

SCOTT, A.D. (1955): "The Fishery: The objectives of Sole Ownership", Journal of Political Economy, 63, 116-124.

SONG A.M., DRESSLER W.H., SATIZÁBAL P. \& FANBINYI M. (2021): "From conversion to conservation to carbon: The changing policy discourse on mangrove governance and use in the Philippines", Journal of Rural Studies, 84, 184-195. D0I: 10.1016/j.jrurstud.2021.01.008. 
STEVENSON, G. (1991): Common Property Economics. A general Theory and Land Use Applications, Cambridge University Press, Cambridge.

STOBBERUP, K., GARZA-GIL, M.D., STIRNEMANN-RELOT, A.R., FRANCESCHELLI, N. \& BLOMEYER, R. (2017): "Small-scale Fisheries and "Blue Growth"' in the EU, 2017 Research for PECH Committee, European Parliament, Policy Department for Structural and Cohesion Policies, Brussels.

SURÍS-REGUEIRO, J.C. \& SANTIAGO, J.L. (2014): “Characterization of fisheries dependence in Galicia (Spain)", Marine Policy, 47, 99-109. DOI: 10.1016/j.marpol.2014.02.006.

TABOADA, M.S. (2004): "El papel de las instituciones en el origen y en la evolución de la cofradía de pescadores gallega. Estudio de casos", Universidad de Santiago de Compostela, Santiago de Compostela.

WILLIAMSON, 0. (2007): "An interview with Oliver Williamson", Journal of Institutional Economics, 3, 3, 373-386. DOI: 10.1017/S1744137407000768.

WORLDFISH, FAO \& DUKE UNIVERSITY (2018): "Illuminating hidden harvests", Penang, Malaysia: WorldFish; Rome, Italy: Food and Agriculture Organization of the United Nations; Durham, USA: Duke University. Program Brief.

\section{Referencias normativas}

Resolución A/RES/70/1, Transformar nuestro mundo: la Agenda 2030 para el Desarrollo Sostenible, 25 de septiembre de 2015, Asamblea General de las Naciones Unidas, 15-16301 (S).

Convención de las Naciones Unidas sobre el Derecho del Mar de 1982, adoptada el 10 de diciembre de 1982, United Nations Treaty Series, Vol. 1833, no 31363.

Reglamento (CE) n² 2371/2002 del Consejo, de 20 de diciembre de 2002, sobre la conservación y la explotación sostenible de los recursos pesqueros en virtud de la política pesquera común, Diario Oficial de la Unión Europea L no 358 de 31 de diciembre de 2002, p. 59/80.

Ley 16/1998, de 25 de junio, de cofradías de pescadores, Boletín Oficial del País Vasco no 129, $12833-12853$.

Ley 3/2001, de 26 de marzo, de Pesca Marítima del Estado", Boletín Oficial del Estado no 75, 11509-11532.

Ley 22/2002, de 12 de julio, de cofradías de pescadores, Diario Oficial de la Generalitat de Cataluña no 3684, 13393-13399. 
Ley 11/2008, de 3 de diciembre, de pesca de Galicia, Boletín Oficial del Estado no 15, 54435506 .

Ley 6/2009, de 11 de diciembre, de modificación de la Ley de pesca de Galicia, Boletín Oficial del Estado no $-30,9816-9834$.

Ley 5/2011, de 29 de marzo, de Economía Social, Boletín Oficial del Estado no 76, 3302333033.

Ley 33/2014, de 26 de diciembre, por la que se modifica la de Pesca Marítima del Estado", Boletín Oficial del Estado no313, 105798-105825.

Ley 6/2016, de 4 de mayo, de la economía social de Galicia, Boletín Oficial del Estado no 147, 42257-42276.

Decreto 152/1991, de 17 de junio, de regulación de las cofradías de pescadores, Diario Oficial de la Generalitat de Cataluña no 1472, 4093-4095.

Decreto 61/1995, de 2 de junio, por el que se regulan las cofradías de pescadores y sus federaciones, Boletín Oficial de las Islas Baleares no 80,6581-6583.

Decreto 145/1995, de 6 de junio, sobre Cofradías de Pescadores y sus Federaciones, Boletín Oficial de la Junta de Andalucía no 92, 6318-6323.

Decreto 86/2004, de 2 de marzo, sobre Cofradías de Pescadores y sus Federaciones, Boletín Oficial de la Junta de Andalucía no 52, 6570-6574.

Decreto 8/2014, de 16 de enero, por el que se regulan las cofradías de pescadores de Galicia y a sus federaciones, Diario Oficial de Galicia no 19, 3629-3696.

Orden de 21 de diciembre de 2020 por la que se aprueba el Plan general de explotación marisquera para el trienio 2021-2023, Diario Oficial de Galicia no 262, 51297-51340. 